\title{
INFLUENCE OF DIPOLE-DIPOLE INTERACTIONS ON STRUCTURAL PROPERTIES OF GAY-BERNE PARTICLES
}

\author{
E. Gwóźdź AND A. BRódKA* \\ Institute of Physics, University of Silesia, Uniwersytecka 4, 40-007 Katowice, Poland
}

\begin{abstract}
Molecular dynamics simulations were performed for a liquid crystal system composed of the Gay-Berne particles with terminal transverse dipole moments. When the dipole-dipole interactions are weak, the isotropic-smectic transition is observed and the transition point shifts towards lower densities as the dipole moment increases. For higher dipole moments the system shows isotropic structure in the whole range of density, and strong dipole--dipole interactions produce two types of dimers that cannot be accommodated into smectic layers.
\end{abstract}

PACS numbers: $31.15 . \mathrm{Qg}, 61.30 . \mathrm{Cz}$

\section{Introduction}

The essential condition for liquid crystallinity is the anisotropy in the molecular shape, however, the presence of dipole moments may affect the transition point and types of phases that are observed. In the last years ellipsoidal Gay-Berne particles [1] with axial dipoles were studied using Monte Carlo simulation method [2-5], and we performed molecular dynamics (MD) simulations of such particles with terminal transverse dipoles $[6,7]$. These studies showed that the dipole-dipole interactions stabilise orientationally ordered phase, i.e. the transition point is shifted towards lower densities or higher temperature compared with the non-polar system.

In this paper we continue our earlier studies and we analyse structural properties of the polar system for several values of the terminal transverse dipole moment.

\footnotetext{
*corresponding author; e-mail: brodka@us.edu.pl
} 


\section{Molecular dynamics simulation}

The behaviour of the liquid crystal system was studied by MD simulation method for 500 molecules in a cubic simulation box. The molecules were modelled by ellipsoidal particles with the terminal transverse dipole moment. In our calculations we used the potential parametrisation as in the original paper by Gay and Berne [1]. In a series of MD simulations, performed for a canonical ensemble, the system was compressed at constant temperature. For the lowest density the molecules were located on the fcc lattice nodes and random orientations as well as velocities were assigned to them. For a larger density the initial configuration was the final configuration of the previous simulation and it was modified decreasing the simulation box size. The other details of the computational procedure have been reported in Refs. [6, 7], and in Table the main parameters of MD simulations are listed only.

TABLE

Parameters of the MD simulation ${ }^{a}$.

\begin{tabular}{l|l}
\hline \hline Dipole moment value position & $\mu^{*}=\mu /\left(4 \pi \varepsilon_{0} \varepsilon_{0} \sigma_{0}^{3}\right)^{1 / 2}=0.5,1.0,1.1,1.2,1.4$ \\
& on the long axis and at distance $\sigma_{0}$ from \\
& the centre of mass \\
Moments of inertia & $I_{\perp}^{*}=I_{\perp} / m \sigma_{0}^{2}=1.0, I_{\|}^{*}=I_{\|} / m \sigma_{0}^{2}=0.2$ \\
Temperature & $T^{*}=k_{\mathrm{B}} T / \varepsilon_{0}=0.60$ \\
Density & $\rho^{*}=\rho \sigma_{0}^{3}=0.10-0.47$ \\
Density increment & $\Delta \rho^{*}=0.01$ \\
Time step & $\Delta t^{*}=\Delta t /\left(m \sigma_{0}^{2} / \varepsilon_{0}\right)^{1 / 2}=0.0015$ \\
Equilibration run & 10000 steps \\
Simulation run & 35000 steps \\
\hline
\end{tabular}

${ }^{a} \varepsilon_{0}$ and $\sigma_{0}$ are units of energy and length and they are the Lennard-Jones potential parameters for the spherical molecule, respectively, $\epsilon_{0}$ is the free space permittivity, $m$ is an arbitrary molecular mass.

\section{Results and discussion}

To characterise orientational order of the particles we calculated an order parameter $P_{2}$ [8], and its density dependence is shown in Fig. 1. For small dipole moments, $\mu^{*} \leq 1.0$, the system exhibits orientationally ordered phase and the isotropic-smectic phase transition point shifts towards lower densities as the dipole moment is increased. For medium dipole, $\mu^{*}=1.1$, partial order of the molecules appears at low densities, however, the maximum value of the $P_{2}$ parameter is lower than that for smaller dipoles. The further increase in the dipole moments, $\mu^{*} \geq 1.2$, causes that the molecules do not exhibit orientational ordering.

Molecular structure and process of orientational ordering are illustrated by the final configurations of the centre of mass positions presented in Fig. 2. For 


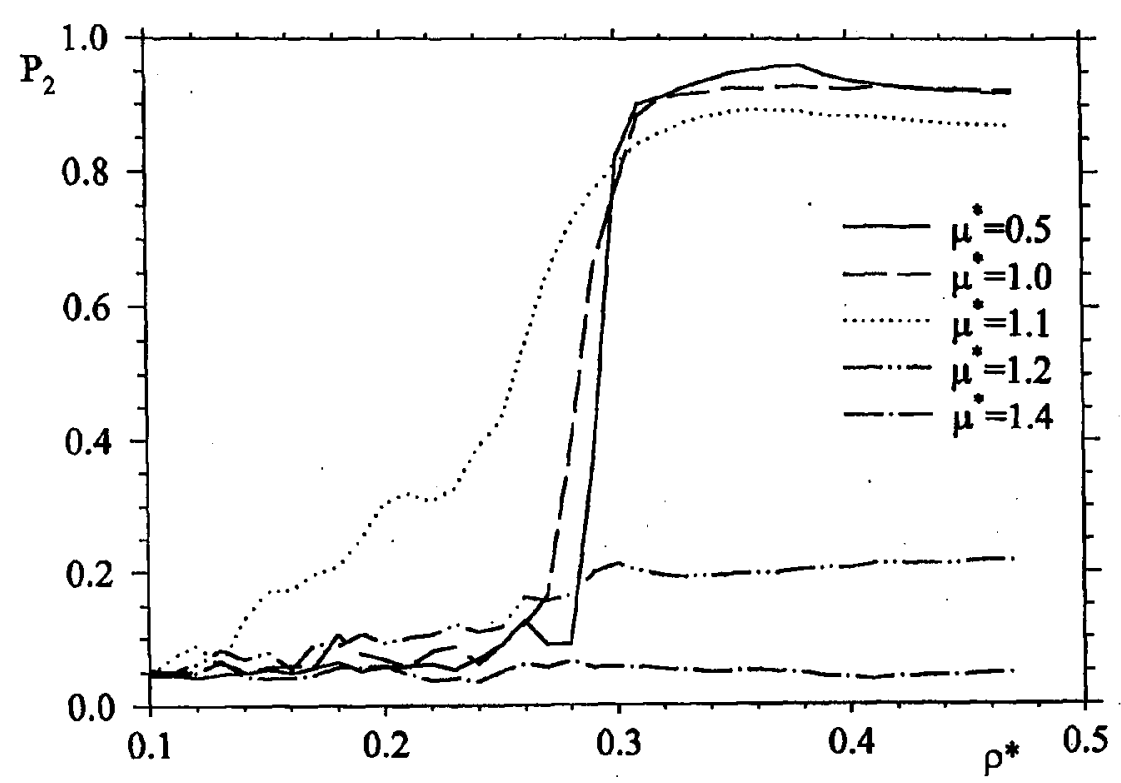

Fig. 1. Density dependence of the orientational order parameter $P_{2}$ for the dipole moments indicated.



Fig. 2. Snapshots of the final positions of the centres of the molecules with (a) $\mu^{*}=0.5$, (b) $\mu^{*}=1.0$, and (c) $\mu^{*}=1.1$ for the densities indicated. The observation direction form an angle $\phi$ with the axis $x$. Open and filled circles indicate molecules with the $z$-axis parallel and antiparallel, respectively, to the vector normal to the smectic plane. 
low dipole moment, $\mu^{*} \leq 1.0$, the system exhibits direct transition to the smectic phase, and in that phase the $z$-axes of the molecules have random antiparallel orientations inside the layers. Initial distribution of the molecules with the medium dipoles, $\mu^{*}=1.1$, is non-homogeneous, which results from strong and attractive dipole-dipole interactions, and the molecules begin to form the layers at relatively low density. Compressing the system the smectic layers are developed but the layers are undulated. The undulation of the smectic layers corresponds to a splay deformation of the director, and hence the order parameter $P_{2}$ is lower than for smaller values of the dipole moment (see Fig. 1). A molecular picture of a splay deformation given by Meyer [9] suggests that the asymmetry of the molecular shape is essential. In our case terminal dipoles introduce strong asymmetry of intermolecular interactions which combined with high density may lead to the undulation of the smectic layers. The $z$-axes of the molecules have essentially the same orientations within the layer. We do not present the configurations of the molecules with higher dipole moment values, $\mu^{*} \geq 1.1$, because the molecules are almost uniformly distributed in the simulation box, however, inhomogeneity of the distribution at low densities is more pronounced than that for $\mu^{*}=1.1$.

To understand behaviour of the molecules with transverse dipole moments we analysed the pair radial distribution functions and orientational correlations of the dipole moments and long molecular axes, but due to the limited scope of this paper we present only conclusions arising from that analysis. One observed that the parallel alignment of dipole pairs is preferred at the nearest neighbour and the molecules form dimers. For small dipoles the dimers have dynamic nature at low densities and they become stable in the smectic phase when two molecules with the closest dipoles has structure (a) presented in Fig. 3. In that sense the dipole-dipole interactions are helpful in orientational ordering of the molecules. For strong dipoles the dipole-dipole interactions dominate and even at low densities the molecules form permanent dimers whose extreme configurations may be identified as structure (b) and (c) in Fig. 3. In other words one has to do with two different kinds of large particles which prevent the system from orientational ordering.


Fig. 3. Configurations of dimers generated by (a) small and medium dipoles, $\mu^{*} \leq 1.1$, and (b), (c) strong ones, $\mu^{*} \geq 1.2$. 


\section{Acknowledgment}

This work was partly supported by the Committee for Scientific Research grant No. 2P03B10317.

\section{References}

[1] J.G. Gay, B.J. Berne, J. Chem. Phys. 74, 3316 (1981).

[2] K. Satoh, S. Mita, S. Kondo, Liq. Cryst. 20, 757 (1996).

[3] K. Satoh, S. Mita, S. Kondo, Chem. Phys. Lett. 255, 99 (1996).

[4] R. Berardi, S. Orlandi, C. Zannoni, Chem. Phys. Lett. 261, 357 (1996).

[5] M. Houssa, A. Oualid, L.F. Rull, Mol. Phys. 94, 439 (1998).

[6] K. Pasterny, E. Gwóźdź, A. Bródka, J. Mol. Liq. 85, 173 (2000).

[7] E. Gwóźdź, A. Bródka, K. Pasterny, J. Mol. Struct., in press.

[8] C. Zannoni, in: The Molecular Physics of Liquid Crystals, Eds. G.R. Luckhurst, G.W. Gray, Academic Press, London 1979, Ch. 9.

[9] R.B. Meyer, Phys. Rev. Lett. 22, 918 (1969). 